\title{
ASYMPTOTIC APPROACH TO THE PERFECT CUBOID PROBLEM
}

\author{
R.A. SHARIPOV
}

\begin{abstract}
The problem of perfect cuboids is one of the old unsolved problems in number theory. By means of various methods it can be reduced to finding a solution of some single Diophantine equation of high degree obeying certain restrictions in the form of inequalities. Each such Diophantine equation is called a characteristic equation of a perfect cuboid. In this paper we present the results obtained by applying asymptotic metods to one of the characteristic equations of a perfect cuboid in the case of the second cuboid conjecture. This results shrink the domain of the integer parameters of the considered characteristic equation and thus make more effective the computer search of perfect cuboids based on this equation.
\end{abstract}

Keywords: perfect cuboid, Diophantine equations, asymptotic methods.

\section{INTRODUCTION}

A perfect cuboid is a rectangular parallelepiped whose edges, the diagonals on the faces, as well as the spatial diagonal are of integer length. The search of perfect cuboids has a long history $[1-50]$. The problem was first mentioned in 1719, but none of cuboids is found yet. The number 1719 by itself is probably not so impressive. It is interesting to compare it with some prominent historical dates: Foundation of Saint-Petersburg (1703), Battle of Poltava (1709), Rebellions of Bashkirs (1735-1740 and 1755), the Celsius scale (1742), births of Mozart (1756) and Beethoven (1770), Pugachev's Rebellion (1773), United States Declaration of Independence (1776), World First Steamship (1783), Great French Revolution (1789), World First Steamship Locomotive (1804), Napoleon Invasion of Russia (1812), Decembrist revolt (1825), opening of the first railway in Russia (1837). In mathematics these are the births of Euler (1707), Lagrange (1736), Fourier (1768), Gauss (1777), Cauchy (1789), Lobachevskii (1792), Abel (1802), Galois (1811). As we see, a lot of events happened in the world which changed essentially our life , but the problem of perfect cuboid was and still remains unsolved. Here we consider one of the approaches to this difficult problem that probably will change the situation.

In paper [51] the problem of constructing a perfect cuboid was reduced to a Diophantine equation of 12-th degree in variables $a, b, u$ and $t$ :

$$
\begin{aligned}
& u^{4} a^{4} b^{4}+6 a^{4} u^{2} b^{4} t^{2}-2 u^{4} a^{4} b^{2} t^{2}-2 u^{4} a^{2} b^{4} t^{2}+4 u^{2} b^{4} a^{2} t^{4}+ \\
& \quad+4 a^{4} u^{2} b^{2} t^{4}-12 u^{4} a^{2} b^{2} t^{4}+u^{4} a^{4} t^{4}+u^{4} b^{4} t^{4}+a^{4} b^{4} t^{4}+ \\
& +6 a^{4} u^{2} t^{6}+6 u^{2} b^{4} t^{6}-8 a^{2} b^{2} u^{2} t^{6}-2 u^{4} a^{2} t^{6}-2 u^{4} b^{2} t^{6}- \\
& \quad-2 a^{4} b^{2} t^{6}-2 b^{4} a^{2} t^{6}+u^{4} t^{8}+b^{4} t^{8}+a^{4} t^{8}+4 a^{2} u^{2} t^{8}+ \\
& +4 b^{2} u^{2} t^{8}-12 b^{2} a^{2} t^{8}+6 u^{2} t^{10}-2 a^{2} t^{10}-2 b^{2} t^{10}+t^{12}=0 .
\end{aligned}
$$

The precise result of paper [51] is formulated in the next theorem.

R.A. Sharipov, Asymptotic approach to the Perfect Cuboid Problem.

(C) SHARIPOV R.A. 2015.

Submitted July 12, 2015. 
Theorem 1.1. A perfect cuboid does exist if and only if Diophantine equation (1.1) has a solution such that $a, b, u, t$ are positive numbers satisfying the inequalities $a<t, b<t, u<t$, $(a+t)(b+t)>2 t^{2}$.

The analysis of equation $(1.1)$ in $[52]$ showed that there are two cases where the polynomial in the left hand side of equation (1.1) is reducible and we can remove inessential factors producing no perfect cuboids. In these cases the degree of equation (1.1) reduces to 8-th and 10-th, respectively. For these cases in [52] the first and the second cuboid conjectures were formulated. For the general case, where no reduction of equation (1.1) does happen, the third cuboid conjecture was formulated.

The first cuboid conjecture $a=b \neq u$. This case turned out to be the simplest one. Despite the first cuboid conjecture remained unproved, in paper [53] it was established that in this case there are no perfect cuboids.

The cases of the second and third cuboid conjectures turned out to be more complicated. For these cases in [54 and [55] only certain structural theorems on integer solutions of equation (1.1) were obtained while the question on whether such solutions do exist and produce perfect cuboids remained open. The second and third conjectures on cuboid also remain neither proved no disproved.

In the present work we consider only the case of the second cuboid conjecture and we summarize the results obtained by the author for this case in eletronic publications [56 59]. On May 19, 2015, the most part of these results was presented at the city seminar on differential equations of mathematical physics named after A.M. Il'in. The author is grateful to the head of the seminar L.A. Kalyakin for the opportuinity to present a talk. The author is grateful to the other head of the seminr V.Yu. Novokshenov and to all participants for the attention and valuable remarks during the talk. The author is also greatefull to B.I. Suleimanov who mentioned the works by A.D. Bruno on Newton polygons and their application in asymptotic analysis.

Papers 60072 made in the time period between papers [51 55 and $[56 \sqrt[59]{56}$ develop an absolutely different approach based on the discrete $S_{3}$-symmetry in the perfect cuboid equations and employing the technique of multi-symmetric polynomials. This approach is planned to be reviewed in a separated publication.

\section{The CASE OF SECOND CONJECTURE OF CUBOID}

The case of the second cuboid conjecture arises when parameters $a, b, u$ in equation 1.1 are related to each other by one of the following two identities:

$$
b u=a^{2} \text {. }
$$

The first of identities (2.1) is resolved by substituting

$$
a=p q, \quad b=p^{2}, \quad u=q^{2} .
$$

The second identity in 2.1 is resolved by substituting

$$
a=p^{2}, \quad b=p q, \quad u=q^{2} .
$$

Applying each of substitutions (2.2) and (2.3) to equation (1.1), we remove an inessential factor and the degree of the equation reduces from 12-th to 10-th. As a result of each of these substitutions the same equation of 10-th degree w.r.t. $p, q$ and $t$ arises:

$$
\begin{aligned}
t^{10} & +\left(2 q^{2}+p^{2}\right)\left(3 q^{2}-2 p^{2}\right) t^{8}+\left(q^{8}+10 p^{2} q^{6}+4 p^{4} q^{4}\right. \\
& \left.-14 p^{6} q^{2}+p^{8}\right) t^{6}-p^{2} q^{2}\left(q^{8}-14 p^{2} q^{6}+4 p^{4} q^{4}+10 p^{6} q^{2}\right. \\
& \left.+p^{8}\right) t^{4}-p^{6} q^{6}\left(q^{2}+2 p^{2}\right)\left(3 p^{2}-2 q^{2}\right) t^{2}-q^{10} p^{10}=0 .
\end{aligned}
$$

Theorem 1.1 implies the following theorem. 
Theorem 2.1. In the case of second cuboid conjecture, a perfect cuboid does exist if and only if Diophantine equation (2.4) has a solution such that $p, q$, $t$ are positive numbers satisfying the inequalities $p q<t, p^{2}<t, q^{2}<t,(p q+t)\left(p^{2}+t\right)>2 t^{2}$.

The second conjecture itself is formulated as follows (see [52]).

Conjecture 2.1 (Second cuboid conjecture). For each two coprime positive integers $p \neq q$, the polynomial of 10-th degree in the left hand side of equation (2.4) is irreducible over the field of raional numbers $\mathbb{Q}$.

Conjecture 2.1 is provided here just to inform the reader and it is not considered in what follows. The main efforts are aimed at the study of equation (2.4).

\section{Motivation of the ASymptotic APproach}

A computer search of perfect cuboids via equation 2.4 is performed iteratively. For instance, like this: for each fixed $p$ we iterate over $q$ and for each $q$ we solve equation (2.4) w.r.t. $t$. Theoretically, for the complete search, for each $p$ we should iterate over all integer values of $q$ from 1 to $+\infty$. In practice, one has to replace $+\infty$ by a fixed large number $q_{\max }(p)$. Equation (2.4) is nice since the choice of the upper bound $q_{\max }(p)$ can be made reasonable and equivalent to the infinite iteration. Roots of polynomial equations of two variables w.r.t. one of the variables demonstrate a rather simple and easily calculable asymptotics as the second variable tend to infinity. Using such asymptotics, in certain cases one can show that for $q$ greater than a certain value $q_{\max }(p)$ equation (2.4) has no integer roots or its integer roots do not satisfy inequalities in Theorem 2.1.

\section{Asymptotics of Roots $t_{i}$ As $q \rightarrow+\infty$}

We denote by $Q_{p q}(t)$ the polynomial in the left hand side of equation (2.4). By this notation we choose variable $t$ as the main one, while variables $p$ and $q$ are regarded as parameters. Polynomial $Q_{p q}(t)$ is even w.r.t. $t$. This is why together with each root $t$, it has the opposite root $-t$. The condition

$$
\begin{cases}t>0, & \text { if } t \text { is a real root, } \\ \operatorname{Im}(t)>0, & \text { if } t \text { is a complex root, }\end{cases}
$$

selects a group of five roots $t_{1}, t_{2}, t_{3}, t_{4}, t_{5}$ of polynomial $Q_{p q}(t)$. The other five roots are obtained by the changing their signs:

$$
t_{6}=-t_{1}, \quad t_{7}=-t_{2}, \quad t_{8}=-t_{3}, \quad t_{9}=-t_{4}, \quad t_{10}=-t_{5} .
$$

The asymptotics of roots of polynomial equations are power $[73$. For a fixed $p=$ const they are written as the series

$$
t_{i}(p, q)=C_{i} q^{\alpha_{i}}\left(1+\sum_{s=1}^{\infty} \beta_{i s} q^{-s}\right) \text { при } q \rightarrow+\infty .
$$

Coefficients $C_{i}$ in $(4.2)$ must be non-zero $C_{i} \neq 0$. These coefficients and the exponents $\alpha_{i}$ can be calculated graphically by means of Newton polygon related with the polynomial $Q_{p q}(t)$ (Fig. 4.1). We write polynomial $Q_{p q}(t)$ formally as

$$
Q_{p q}(t)=\sum_{m=0}^{10} \sum_{r=0}^{10} A_{m r}(p) q^{r} t^{m} .
$$

The number of terms in formal sum 4.3 is 121. The actual number of terms in polynomial $Q_{p q}(t)$ is much less. Drawing the Newton polygon, one chooses non-zero terms in 4.3 and they are marked as the points with coordinates $(m, r)$ on the coordinate plane. Since coordinates $(m, r)$ are integer, these points are the sites of the integer lattice (Fig. 4.1). 
Definition 4.1. For each polynomial of two variables $P(t, q)$, the convex hull of all sites of the integer lattice associated with its monomials is called the Newton polygon of this polynomial $P(t, q)$.

The boundary of the Newton polygon shown in Figure 4.1 consists of two parts, the upper one and the lower one. The upper part of the boundary contains the sites $A_{010}, A_{210} A_{410}, A_{68}$ $A_{84}$ and $A_{100}$. The associated coefficients of polynomial $Q_{p q}(t)$ are given by the formulae

$$
\begin{array}{lll}
A_{010}=-p^{10}, & A_{210}=2 p^{6}, & A_{410}=-p^{2}, \\
A_{68}=1, & A_{84}=6, & A_{100}=1 .
\end{array}
$$

The following theorem expresses a well-known fact. Its proof can be found in [56].

Theorem 4.1. Exponents $\alpha_{i}$ in asymptotic expansions 4.2 are calculated by the formula $\alpha_{i}=-k$, where $k$ is the slope of the segments of the polyline being the upper boundary of the Newton polygon in Figure 4.1.

In our case Theorem 4.1 gives the following possible values for exponents $\alpha_{i}$ in asymptotic expansions 4.2):

$$
\alpha_{i}=0, \quad \alpha_{i}=1, \quad \alpha_{i}=2 .
$$

The number of the roots satisfying condition (4.1) exceeds three. This is why some of roots $t_{1}$, $t_{2}, t_{3}, t_{4}, t_{5}$ have the same growth rate as $q \rightarrow+\infty$.

Besides $\alpha_{i}$, each of roots $t_{1}, t_{2}, t_{3}, t_{4}, t_{5}$ is characterized by its coefficient $C_{i}$ in expansions (4.2). These coefficients are also calculated by means of the Newton polygon. 
The case $\alpha_{i}=0$ in 4.5 corresponds to the horizontal segment on the upper boundary of the Newton polygon. On this segment there three sites $A_{410}, A_{210}$, and $A_{010}$. This is why the corresponding equation for $C_{i}$ reads as

$$
A_{410} C_{i}^{4}+A_{210} C_{i}^{2}+A_{010}=0 .
$$

In view of (4.4), equation (4.6) has two real roots

$$
C_{i}=p^{2}, \quad C_{i}=-p^{2},
$$

each being of multiplicity 2. Condition (4.1) excludes the root $C_{i}=-p^{2}$ in (4.7), keeping only one double root. The associated expansion is

$$
t_{i}(p, q)=p^{2}\left(1+\sum_{s=1}^{\infty} \beta_{i s} q^{-s}\right) .
$$

Case $\alpha_{i}=1$ in 4.5 corresponds to the short sloping segment on the uppoer boundary of Newton polygon. This segment contains two sites $A_{410}$ and $A_{68}$. The associated coefficient $C_{i}$ in 4.2 is determined by the equation

$$
A_{68} C_{i}^{6}+A_{410} C_{i}^{4}=0 .
$$

In view of (4.4) and $C_{i} \neq 0$, equation 4.9 has two simple real roots:

$$
C_{i}=p, \quad C_{i}=-p .
$$

Condition (4.1) excludes the root $C_{i}=-p$ in 4.10 keeping only one root $C_{i}=p$. The expansion associated with this root reads as

$$
t_{i}(p, q)=p q\left(1+\sum_{s=1}^{\infty} \beta_{i s} q^{-s}\right)
$$

Case $\alpha_{i}=2$ in 4.5 corresponds to a long sloping segment on the upper boundary of Newton polygon. This segment contains three sites $A_{68}, A_{84}$ and $A_{100}$. This is why the corresponding equation for $C_{i}$ reads as

$$
A_{100} C_{i}^{10}+A_{84} C_{i}^{8}+A_{68} C_{i}^{6}=0 .
$$

In view of 4.4 and $C_{i} \neq 0$, equation 4.12 has four complex roots:

$$
\begin{aligned}
C_{i} & =(\sqrt{2}+1) \mathfrak{i}, & C_{i} & =(\sqrt{2}-1) \mathfrak{i}, \\
C_{i} & =-(\sqrt{2}+1) \mathfrak{i}, & C_{i} & =-(\sqrt{2}-1) \mathfrak{i} .
\end{aligned}
$$

Here $\mathfrak{i}=\sqrt{-1}$. Roots 4.14 are excluded by condition 4.1. Only two roots 4.13 are kept and they give the asymptotic expansions:

$$
t_{i}(p, q)=(\sqrt{2}+1) \mathfrak{i} q^{2}\left(1+\sum_{s=1}^{\infty} \beta_{i s} q^{-s}\right), \quad t_{i}(p, q)=(\sqrt{2}-1) \mathfrak{i} q^{2}\left(1+\sum_{s=1}^{\infty} \beta_{i s} q^{-s}\right) .
$$

Formulae 4.8, 4.11), 4.15) are summarized by the following theorem.

Theorem 4.2. For sufficiently large $q$, i.e., as $q>q_{\text {min }}$, equation (2.4) of 10-th degree has five simple roots satisfying condition (4.1). Three of them $t_{1}, t_{2}, t_{3}$ are real. Their asymptotics as $q \rightarrow+\infty$ are given by the formulae

$$
t_{1} \sim p^{2}, \quad t_{2} \sim p^{2}, \quad t_{3} \sim p q .
$$

Other two roots of equation (2.4) are complex-valued. The asymptotics of these roots as $q \rightarrow+\infty$ are described by the formulae

$$
t_{4} \sim(\sqrt{2}+1) \mathfrak{i} q^{2}, \quad t_{5} \sim(\sqrt{2}-1) \mathfrak{i} q^{2} .
$$




\section{ASYMPtotic ESTimate FOR REAL ROOTS}

Asymptotic expansions (4.8), 4.11), 4.15) and formulae (4.16), (4.17) describe the limiting behavior of the roots to equation (2.4) as $q \rightarrow+\infty$. They give no precise information on location of roots for finite $q$. In order to have such information, we need asymptotic estimates, namely, the estimates for the error terms for finite sums of asymptotic series.

In accordance with formulae (4.16), roots $t_{1}$ and $t_{2}$ do not grow as $q \rightarrow+\infty$. This is why their asymptotic series (4.8) can be truncated right after the leading term and we can write formula 4.8 as the following sums:

$$
t_{1}=p^{2}+R_{1}(p, q), \quad t_{2}=p^{2}+R_{2}(p, q) .
$$

Our next aim is to obtain the estimates

$$
\left|R_{i}(p, q)\right|<\frac{C(p)}{q}, \text { where } i=1,2 .
$$

To achieve this aim, we substitute

$$
t=p^{2}+\frac{c}{q}
$$

into equation (2.4). In the obtained equation we make one more substitution

$$
q=\frac{1}{z}
$$

After two substitutions (5.3) and (5.4) and after removing the denominators equation (2.4) becomes a new polynomial equation in new variables $c$ and $z$. A feature of this equation is that it is written as

$$
16 p^{12}+f(c, p, z)=4 p^{6} c^{2} .
$$

Here $f(c, p, z)$ is a polynomial given by an explicit formula. But the formula for $f(c, p, z)$ is bulky. It is written in a machine-readable formate and is provided in the ancillary file strategy_formulas.txt attached to electronic publication [56. The link for downloading this file is provided on the site http://arXiv.org/abs/1504.07161.

Polynomial $f(c, p, z)$ vanishes at $z=0$. This is why its values are sufficiently small for small $z$. Let $q \geqslant 59 p$ and parameter $c$ range the interval

$$
-5 p^{3}<c<0
$$

By $q \geqslant 59 p$ and (5.4) we obtain the estimate $|z| \leqslant 1 / 59 p^{-1}$. Employing this estimate and inequalities (5.6), by straightforward calculations we can get the following estimate:

$$
|f(c, p, z)|<15 p^{12}
$$

For fixed $p$ and $z$ estimate (5.7) means that the left hand side of equation (5.5) is a continuous function of $c$ ranging from $p^{12}$ to $31 p^{12}$ as $c$ ranges in interval (5.6). The right hand side of equation (5.5) is also a continuous function of $c$. It decreases monotonically from $100 p^{12}$ to 0 on interval (5.6). Hence, interval (5.6) contains at least one root of equation (5.5).

Parameter $c$ is related with variable $t$ by formula (5.3). Inequalities (5.5) for $c$ imply the following inequalities for $t$ :

$$
p^{2}-\frac{5 p^{3}}{q}<t<p^{2}
$$

Inequalities (5.8) and the above arguments give the following theorem.

Theorem 5.1. For each $q \geqslant 59 p$ equation (2.4) has at least one real root satisfying inequalities (5.8). 
The above arguments can be reproduced for the case when parameter $c$ ranges the interval mirror-symmetric to interval (5.6):

$$
0<c<5 p^{3}
$$

In this case $(5.9)$ and $(5.3)$ imply the inequalities

$$
p^{2}<t<p^{2}+\frac{5 p^{3}}{q}
$$

for variable $t$ and we obtain the following theorem.

Theorem 5.2. For each $q \geqslant 59 p$ equation (2.4) has at least one real root satisfying inequalities (5.10).

Theorems 5.1 and 5.2 provide asymptotic estimates $(5.2)$ with $C(p)=5 p^{3}$ for error terms $R_{1}(p, q)$ and $R_{2}(p, q)$ in formulae (5.1).

In accordance with formulae (4.16), root $t_{3}$ grows as $q \rightarrow+\infty$. We write the asymptotic formula with an error term for this root:

$$
t_{3}=p q-\frac{16 p^{3}}{q}+R_{3}(p, q)
$$

and seek an estimate for error term $R_{3}(p, q)$ as

$$
\left|R_{3}(p, q)\right|<\frac{C(p)}{q^{2}}
$$

We then write inequalities

$$
p q-\frac{16 p^{3}}{q}-\frac{5 p^{4}}{q^{2}}<t<p q-\frac{16 p^{3}}{q}+\frac{5 p^{4}}{q^{2}}
$$

and formulate the following theorem.

Theorem 5.3. For each $q \geqslant 59 p$ equation (2.4) has at least one real root satisfying inequalities (5.13).

The proof of Theorem 5.3 can be found in [56]. It is almost the same as the proof of Theorems 5.1 and 5.2 given above.

Inequalities (5.13) prove estimate 5.12 with $C(p)=5 p^{4}$ for error term $R_{3}(p, q)$ in asymptotic formula 5.11 .

\section{Asymptotic eSTIMATES FOR COMPLEX ROOTS}

The complexity of roots $t_{4}$ and $t_{5}$ is implied by formulae 4.15 . For complex root $t_{4}$ we write the asymptotic formula

$$
t_{4}=(\sqrt{2}+1) \mathfrak{i} q^{2}+(\sqrt{2}-2) \mathfrak{i} p^{2}+R_{4}(p, q), \text { where } \mathfrak{i}=\sqrt{-1} .
$$

For error term $R_{4}(p, q)$ we seek an estimate

$$
\left|R_{4}(p, q)\right|<\frac{C(p)}{q}
$$

We write the inequalities

$$
(\sqrt{2}+1) q^{2}+(\sqrt{2}-2) p^{2}-\frac{5 p^{3}}{q}<\operatorname{Im} t<(\sqrt{2}+1) q^{2}+(\sqrt{2}-2) p^{2}+\frac{5 p^{3}}{q}
$$

and formulate the following theorem.

Theorem 6.1. For each $q \geqslant 59 p$ equation (2.4) has at least one pure imaginary root satisfying inequalities (6.3). 
Inequalities (6.3) prove estimate $(6.2)$ with $C(p)=5 p^{3}$ for the error term in asymptotic formula 6.1).

Theorem 6.1 is similar to Theorems 5.1, 5.2, 5.3. Its proof is provided in 556.

Complex root $t_{5}$ of equation (2.4) is similar to root $t_{4}$. For complex root $t_{5}$ we write asymptotic formula

$$
t_{4}=(\sqrt{2}-1) \mathfrak{i} q^{2}+(\sqrt{2}+2) \mathfrak{i} p^{2}+R_{5}(p, q), \text { where } \mathfrak{i}=\sqrt{-1} .
$$

For error term $R_{5}(p, q)$ we seek estimate

$$
\left|R_{5}(p, q)\right|<\frac{C(p)}{q}
$$

We write the inequalities

$$
(\sqrt{2}-1) q^{2}+(\sqrt{2}+2) p^{2}-\frac{5 p^{3}}{q}<\operatorname{Im} t<(\sqrt{2}-1) q^{2}+(\sqrt{2}+2) p^{2}+\frac{5 p^{3}}{q}
$$

and formulate the following theorem.

Theorem 6.2. For each $q \geqslant 59 p$ equation (2.4) has at least one pure imaginary root satisfying inequalities (6.6).

Inequalities (6.6) prove estimate (6.5) with $C(p)=5 p^{3}$ for error term in asymptotic formula 6.4. The proofs of inequality (6.6) and Theorem 6.2 can be found in work [56].

\section{ASYMPTOTIC INTERVALS}

Inequalities (5.8), 5.10, (5.13), 6.3), 6.6) define five open intervals. The first three of them are on the real axis. The other two are on the imaginary axis. Several pure technical results on asymptotic intervals (5.8), (5.10), (5.13), (6.3), (6.6) were proven in [56]. The essence of these technical results is that as $q \geqslant 59 p$, the asymptotic intervals are mutually disjoint. The first three of them are on the positive part of the real axis, and the other two are on the positive part of the imaginary axis. Along with Theorems 5.1, 5.2, 5.3, 6.1, 6.2 it leads us to the following result.

Theorem 7.1. For $q \geqslant 59 p$, five roots $t_{1}, t_{2}, t_{3}, t_{4}, t_{5}$ of equation (2.4) satisfying conditions (4.1) are simple. They are located in five mutually disjoint intervals (5.8), (5.10), (5.13), (6.3), (6.6) so that each interval contains exactly one root.

\section{INTEGER POINTS OF ASYMPTOTIC INTERVALS}

In view of formulae (5.8), (5.10), (5.13), (6.3), (6.6) it is easy to see that the lengths of asymptotic intervals tend to zero as $q \rightarrow+\infty$. It naturally lowers the chance of integer points to be in these intervals. The following theorems were proven in work [56].

Theorem 8.1. For $q \geqslant 59 p$ and $q>5 p^{3}$ asymptotic intervals (5.8) and (5.10) contain no integer points.

Theorem 8.2. For $q \geqslant 59 p$ and $q^{2}>10 p^{4}$ asymptotic interval (5.13) contains at most one integer point.

Theorem 8.3. For $q \geqslant 59 p$ and $q \geqslant 16 p^{3}+5 p / 16$ asymptotic interval 5.13) contains no integer points.

We note that in accordance with Theorem 2.1, not each integer solution to equation 2.4 produces a perfect cuboid. In addition, the inequalities

$$
p q<t, \quad p^{2}<t, \quad q^{2}<t, \quad(p q+t)\left(p^{2}+t\right)>2 t^{2} .
$$

should be satisfied. 
Belonging of the roots to equation $(2.4)$ to asymptotic intervals $(5.8),(5.10),(5.13),(6.3)$, (6.6) as $q \geqslant 59 p$ produces new inequalities for them. By comparing inequalities (8.1) with inequalities (5.8), (5.10), (5.13), it was shown in [56] that for $q \geqslant 59 p$ the integer points in the real asymptotic intervals did not satisfy inequalities 8.1). It implied the main result of work [56].

Theorem 8.4. For $q \geqslant 59 p$ Diophantine equation (2.4) has no solutions producing perfect cuboids.

For each fixed $p$ Theorem 8.4 provides the upper bound $q_{\max }(p)=59 p$, up to which one should perform a numerical search of perfect cuboids in the case of second cuboid conjecture.

\section{REVERSE ASYMPTOTICS}

In [57], parameters $p$ and $q$ of equation (2.4) switch their roles. In this paper parameter $q$ is kept fixed, while parameter $p$ tends to infinity. The asymptotics obtained under these conditions were called reverse. A substantial part of the results of [56] was extendedto the case of reverse asymptotics. Namely, the asymptotic expansions with asymptotic estimates were found, as well as asymptotic intervals for roots $t_{1}, t_{2}, t_{3}, t_{4}, t_{5}$ of equation (2.4) satisfying conditions (4.1). These asymptotic intervals are defined by the following inequalities:

$$
\begin{aligned}
& p q+\frac{16 q^{3}}{p}-\frac{5 q^{4}}{p^{2}}<t<p q+\frac{16 q^{3}}{p}+\frac{5 q^{4}}{p^{2}} \\
& p^{2}-2 q p-2 q^{2}-\frac{9 q^{3}}{p}<t<p^{2}-2 q p-2 q^{2} \\
& p^{2}+2 q p-2 q^{2}<t<p^{2}+2 q p-2 q^{2}+\frac{9 q^{3}}{p}, \\
& (\sqrt{2}+1) q^{2}-\frac{5 q^{3}}{p^{2}}<\operatorname{Im} t<(\sqrt{2}+1) q^{2}+\frac{5 q^{3}}{p^{2}}, \\
& (\sqrt{2}-1) q^{2}-\frac{5 q^{3}}{p^{2}}<\operatorname{Im} t<(\sqrt{2}-1) q^{2}+\frac{5 q^{3}}{p^{2}} .
\end{aligned}
$$

An analogue of Theorem 7.1 is the following theorem proven in [57].

Theorem 9.1. For $p \geqslant 59 q$ five roots $t_{1}, t_{2}, t_{3}, t_{4}, t_{5}$ to equation (2.4) satisfying conditions (4.1) are simple. They are located in five mutually disjoint intervals (9.1), (9.2), (9.3), (9.4), (9.5) such that each interval contains one root.

It is easy to see that the lengths of asymptotic intervals tend to zero as $p \rightarrow+\infty$. Employing this fact, in [57] the following three theorems were formulated.

Theorem 9.2. For $p \geqslant 59 q$ and $p>9 q^{3}$ asymptotic intervals (9.2) and (9.3) contain no integer points.

Theorem 9.3. For $p \geqslant 59 q$ and $p^{2}>10 q^{4}$ asymptotic interval (9.1) contains at most one integer point.

Theorem 9.4. For $p \geqslant 59 q$ and $p \geqslant 16 q^{3}+5 q / 16$ asymptotic interval (9.1) contains no integer points.

Theorems 9.2, 9.3, 9.4 are analogues of Theorems 8.1, 8.2, 8.3. Their proofs reproduce almost literally the proof of Theorems 8.1, 8.2, 8.3 provided in [56]. This is why it was not given in [57] and is not provided here.

Despite of essential likeness, the parallel between papers [56] and [57] is not complete. We did not succeed to prove an analogue of Theorem 8.4 in [57]. Instead of this, a weaker result is obtained. It is provided in the following theorem. 
Theorem 9.5. Under the condition $p \geqslant 59 q$ and the condition $p>9 q^{3}$ Diophanite equation (2.4) has no solutions producing perfect cuboids.

Summarizing the results of Theorems 8.4 and 9.5 , in [57] three regions in the positive quadrant of the coordinate $p q$-plane were defined:

1. Linear region defined by linear inequalities

$$
\frac{q}{59}<p, \quad p<59 q
$$

2. Nonlinear region defined by nonlinear inequalities

$$
59 q \leqslant p, \quad p \leqslant 9 q^{3}
$$

3. No cuboids region, which includes all points outside the first two regions.

\section{ASYMPtotics AS BOth $p$ AND $q$ SimultaneOUSLY TEND TO INFINITY}

In [58] and [59], the asymptotics for the roots to equation (2.4) were considered as both parameters $p$ and $q$ simultaneously tend to infinity. The main result of [58] is based on the asymptotics where

$$
p-q=\text { const } .
$$

This result can be formulated as the following theorem.

Theorem 10.1. If positive integer parameters $p$ and $q$ of equation (2.4) satisfy the inequalities

$$
q-\frac{q}{97} \leqslant p, \quad p \leqslant q+\min \left(\frac{q}{97}, \sqrt[3]{\frac{q}{74}}\right)
$$

then equation (2.4) has no solutions producing perfect cuboids.

The main result of [59] is based on the asymptotics as

$$
p-B q^{3}=\text { const } \text { and } B=1,2, \ldots, 9 .
$$

Here, instead of (10.1), the inequalities

$$
\begin{gathered}
B q^{3}-\frac{q^{3}}{3600^{3}} \leqslant p \leqslant B q^{3}+\frac{q^{3}}{3600^{3}}, \\
B q^{3}-2 q<p<B q^{3}+2 q,
\end{gathered}
$$

arise and the following theorems are formulated.

Theorem 10.2. For each $B=1,2, \ldots, 9$ except $B=5$, if inequalities 10.2) are satisfied, then equation (2.4) has no solutions producing perfect cuboids.

Theorem 10.3. As $B=5$, if both inequalities 10.2 and 10.3 are satisfied, then Dipohantine equation (2.4) has no solutions producing perfect cuboids.

The results stated in Theorem 10.1 and Theorems 10.2, 10.3 are of a restricted nature. By means of (10.1), 10.2 and 10.3 they specify small subdomains in the linear and nonlinear regions defined by inequalities (9.6) and (9.7) above and ensure the absence of perfect cuboids in the specified subdomains. 


\section{FURTHER PERSPECTIVES}

Until the problem of perfect cuboids is solved, nobody can say in which way its solution will be found. Concerning the asymptotic approach to the problem in the case of the second cuboid conjecture, the author believes that it is promising to consider invertible transformations of parameters

$$
\tilde{p}=\tilde{p}(p, q), \quad \tilde{q}=\tilde{q}(p, q),
$$

see $[74]$, and then to study the asymptotics as $\tilde{q} \rightarrow+\infty$ and $\tilde{p}=$ const. It is also possible to study the asymptotics as $p$ and $q$ tend independently to infinity by employing the techniques of Newton polyhedra developed in numerous works by Bruno, Arnold, Bernstein, Varchenko, Volevich, Gindikin, Kushnerenko, Khovansky, Soleev and other. This approach is more complicated since it will take some time to learn the above mentioned technique.

\section{BIBLIOGRAPHY}

1. P. Halcke. Deliciae mathematicae oder mathematisches Sinnen-Confect. N. Sauer, Hamburg (1719).

2. N. Saunderson. Elements of algebra. 2. Cambridge Univ. Press, Cambridge (1740).

3. L. Euler. Vollständige Anleitung zur Algebra, Kayserliche Akademie der Wissenschaften, St. Petersburg (1771).

4. H.C. Pocklington. Some Diophantine impossibilities // Proc. Cambridge Phil. Soc. 17, 108-121 (1912).

5. L.E. Dickson. History of the theory of numbers. V. 2: Diophantine analysis. Dover, New York (2005).

6. M. Kraitchik. On certain rational cuboids // Scripta Math. 11, 317-326 (1945).

7. M. Kraitchik. Théorie des Nombres. Tome 3. Analyse Diophantine et application aux cuboides rationelles. Gauthier-Villars, Paris (1947).

8. M. Kraitchik. Sur les cuboides rationelles // Proc. Int. Congr. Math., Amsterdam. 2, 33-34 (1954).

9. T.B. Bromhead. On square sums of squares // Math. Gazette. 44:349, 219-220 (1960).

10. M. Lal, W.J. Blundon. Solutions of the Diophantine equations $x^{2}+y^{2}=l^{2}, y^{2}+z^{2}=m^{2}$, $z^{2}+x^{2}=n^{2} / /$ Math. Comp. 20:93, 144-147 (1966).

11. W.G. Spohn. On the integral cuboid // Amer. Math. Monthly. 79:1, 57-59 (1972).

12. W.G. Spohn. On the derived cuboid // Canad. Math. Bull. 17:4, 575-577 (1974).

13. E.Z. Chein. On the derived cuboid of an Eulerian triple // Canad. Math. Bull. 20:4, 509-510 (1977).

14. J. Leech. The rational cuboid revisited // Amer. Math. Monthly. 84:7, 518-533 (1977); Erratum // Amer. Math. Monthly. 85:6, 472 (1978).

15. J. Leech. Five tables relating to rational cuboids // Math. Comp. 32:142, 657-659 (1978).

16. W. G. Spohn. Table of integral cuboids and their generators // Math. Comp. 33:145, 428-429 (1979).

17. J. Lagrange. Sur le dérivé du cuboide Eulérien // Canad. Math. Bull. 22:2, 239-241 (1979).

18. J. Leech. A remark on rational cuboids // Canad. Math. Bull. 24:3, 377-378 (1981).

19. I. Korec. Nonexistence of small perfect rational cuboid // Acta Math. Univ. Comen. 42/43, 73-86 (1983).

20. I. Korec. Nonexistence of small perfect rational cuboid II // Acta Math. Univ. Comen. 44/45, 39-48 (1984).

21. D.G. Wells. The Penguin dictionary of curious and interesting numbers, Penguin publishers, London (1986).

22. A. Bremner, R.K. Guy. A dozen difficult Diophantine dilemmas // Amer. Math. Monthly. 95:1, 31-36 (1988).

23. A. Bremner. The rational cuboid and a quartic surface// Rocky Mountain J. Math. 18:1, 105-121 (1988). 
24. W.J.A. Colman., On certain semiperfect cuboids // Fibonacci Quart. 26:1, 54-57 (1988); Some observations on the classical cuboid and its parametric solutions // Fibonacci Quart. 26:4, 338-343 (1988).

25. I. Korec. Lower bounds for perfect rational cuboids // Math. Slovaca. 42:5, 565-582 (1992).

26. R.K. Guy. Is there a perfect cuboid? Four squares whose sums in pairs are square. Four squares whose differences are square // In book "Unsolved Problems in Number Theory", 2-nd ed., Springer-Verlag, New York, 173-181 (1994).

27. R.L. Rathbun, T. Granlund. The integer cuboid table with body, edge, and face type of solutions // Math. Comp. 62:205, 441-442 (1994).

28. R.L. Rathbun, T. Granlund. The classical rational cuboid table of Maurice Kraitchik // Math. Comp. 62:205, 442-443 (1994).

29. W.J.A. Colman. A perfect cuboid in Gaussian integers // Fibonacci Quart. 32:3, 266-268 (1994).

30. B.E. Peterson, J.H. Jordan. Integer hexahedra equivalent to perfect boxes // Amer. Math. Monthly. 102:1, 41-45 (1995).

31. R. Van Luijk. On perfect cuboids. Doctoraalscriptie, Mathematisch Instituut, Universiteit Utrecht, Utrecht (2000).

32. F. Luca. Perfect cuboids and perfect square triangles // Math. Magazine. 73:5, 400-401 (2000).

33. R.L. Rathbun. The rational cuboid table of Maurice Kraitchik // Preprint: arXiv:math.HO/0111229.

34. N. Narumiya, H. Shiga. On Certain Rational Cuboid Problems // Nihonkai Math. Journal. 12:1, 75-88 (2001).

35. R. Hartshorne, R. Van Luijk. Non-Euclidean Pythagorean triples, a problem of Euler, and rational points on K3 surfaces // The Mathematical Intelligencer. 30:4, 4-10 (2008).

36. Waldschmidt M., Open diophantine problems // Mosc. Math. J. 4:1, 245-305 (2004).

37. E.J. Ionascu, F. Luca, P. Stanica. Heron triangles with two fixed sides // J. Number Theory. 126:1, 52-67 (2007).

38. A. Ortan, V. Quenneville-Belair. Euler's brick // Delta Epsilon, McGill Undergraduate Mathematics J. 1, 30-33 (2006).

39. O. Knill. Hunting for Perfect Euler Bricks // Harvard College Math. Review. 2:2, 102 (2008).

40. N.J.A. Sloan. Sequences A031173 (http://oeis.org/A031173), A031174 (http://oeis.org /A03 1174), and A031175 (http://oeis.org/A031175), On-line encyclopedia of integer sequences, OEIS Foundation Inc., Portland, USA.

41. T.S. Roberts. Some constraints on the existence of a perfect cuboid // Australian Math. Soc. Gazette. 37:1, 29-31 (2010).

42. M. Stoll, D. Testa. The surface parametrizing cuboids // Preprint: arXiv:1009.0388.

43. J. Sawyer, C.A. Reiter. Perfect parallelepipeds exist// Math. Comp. 80:274, 1037-1040 (2011).

44. M. Meskhishvili. Perfect cuboid and congruent number equation solutions // Preprint: arXiv: 1211.6548 .

45. A. Beauville. A tale of two surfaces // Preprint: arXiv:1303.1910.

46. E. Freitag, R.S. Manni. Parametrization of the box variety by theta functions // Preprint: arXiv:1303.6495.

47. B.D. Sokolowsky, R.M. VanHooft, C.A. Reiter. An infinite family of perfect parallelepipeds // Math. Comp. 83:289, 2441-2454 (2014).

48. M. Meskhishvili. Parametric solutions for a nearly-perfect cuboid // Preprint: arXiv:1502.02 375.

49. S. Kitchen. On the existence of perfect cuboids // OURE publication, Missouri University of Science and Technology (2015).

50. W. Wyss. On perfect cuboids // Preprint: arXiv:1506.02215.

51. R.A. Sharipov. A note on a perfect Euler cuboid // Preprint: arXiv:1104.1716.

52. R.A. Sharipov. Perfect cuboids and irreducible polynomials // Ufimskij Matem. Zhurn. 4:1, 153160 (2012). [Ufa Math. J. 4:1, 143-150 (2012).]

53. R.A. Sharipov. A note on the first cuboid conjecture // Preprint: arXiv:1109.2534.

54. R.A. Sharipov. A note on the second cuboid conjecture. Part I// Preprint: arXiv:1201.1229.

55. R.A. Sharipov. A note on the third cuboid conjecture. Part I // Preprint: arXiv:1203.2567. 
56. A.A. Masharov, R.A. Sharipov. A strategy of numeric search for perfect cuboids in the case of the second cuboid conjecture // Preprint: arXiv:1504.07161.

57. R.A. Sharipov. Reverse asymptotic estimates for roots of the cuboid characteristic equation in the case of the second cuboid conjecture // Preprint: arXiv:1505.00724.

58. R.A. Sharipov. Asymptotic estimates for roots of the cuboid characteristic equation in the linear region // Preprint: arXiv:1505.02745.

59. R.A. Sharipov. Asymptotic estimates for roots of the cuboid characteristic equation in the nonlinear region // Preprint: arXiv:1506.04705.

60. R.A. Sharipov. Perfect cuboids and multisymmetric polynomials // Preprint: arXiv:1205.3135.

61. R.A. Sharipov. On an ideal of multisymmetric polynomials associated with perfect cuboids // Preprint: arXiv:1206.6769.

62. R.A. Sharipov. On the equivalence of cuboid equations and their factor equations // Preprint: arXiv:1207.2102.

63. R.A. Sharipov. A biquadratic Diophantine equation associated with perfect cuboids // Preprint: arXiv:1207.4081.

64. J.R. Ramsden. A general rational solution of an equation associated with perfect cuboids // Preprint: arXiv:1207.5339.

65. J.R. Ramsden, R.A. Sharipov. Inverse problems associated with perfect cuboids // Preprint: arXiv:1207.6764.

66. R.A. Sharipov. On a pair of cubic equations associated with perfect cuboids // Preprint: arXiv:1208.0308.

67. R.A. Sharipov. On two elliptic curves associated with perfect cuboids // Preprint: arXiv: 1208.1227.

68. J.R. Ramsden, R.A. Sharipov. On singularities of the inverse problems associated with perfect cuboids // Preprint: arXiv:1208.1859.

69. J.R. Ramsden, R.A. Sharipov. On two algebraic parametrizations for rational solutions of the cuboid equations // Preprint: arXiv:1208.2587.

70. R.A. Sharipov. A note on solutions of the cuboid factor equations // Preprint: arXiv:1209.0723.

71. R.A. Sharipov. A note on rational and elliptic curves associated with the cuboid factor equations // Preprint: arXiv:1209.5706.

72. J.R. Ramsden, R.A. Sharipov. Two and three descent for elliptic curves associated with perfect cuboids // Preprint: arXiv:1303.0765.

73. I.S. Kaschenko. Asymptotic expansions of solutions to equations, Yaroslavl State Univ., Yaroslavl (2011). (in Russian).

74. R.A. Sharipov. A note on invertible quadratic transformations of the real plane // Preprint: arXiv:1507.01861.

Ruslan Abdullovich Sharipov, Bashkir State University,

Zaki Validi, 32, 450074, Ufa, Russia

E-mail: $r$-sharipov@mail.ru 